\title{
Quantitative Analysis in Multimodality Imaging: Challenges and Opportunities
}

\section{Type of article: conference Abstract}

\author{
Habib Zaidi \\ Geneva University Hospital, Switzerland \\ Email: habib.zaidi@hcuge.ch Web: http://pinlab.hcuge.ch
}

\begin{abstract}
This talk reflects the tremendous ongoing interest in molecular and dual-modality imaging (PET/CT, SPECT/CT and PET/MR) as both clinical and research imaging modalities in the past decade. An overview of molecular multi-modality medical imaging instrumentation as well as simulation, reconstruction, quantification, and related image processing issues with special emphasis on quantitative analysis of nuclear medical images are presented. This tutorial aims to bring the biomedical image processing community a review on the state-of-the-art algorithms used and under development for accurate quantitative analysis in multimodality and multiparametric molecular imaging and their validation mainly from the developer's perspective with emphasis on image reconstruction and analysis techniques. It will inform the audience about a series of advanced development recently carried out at the PET instrumentation \& Neuroimaging Lab of Geneva University Hospital and other active research groups. Current and prospective future applications of quantitative molecular imaging also are addressed, especially its use prior to therapy for dose distribution modeling and optimization of treatment volumes in external radiation therapy and patient-specific 3D dosimetry in targeted therapy toward the concept of image-guided radiation therapy.
\end{abstract}

Keywords: Image processing, Multimodality imaging, nuclear medical images, PET/CT, SPECT/CT, PET/MR, Quantitative analysis.

\section{Declaration of conflicts}

This article was presented at ICHSMT'16 as a key notes.

\section{Authors' biography}

Habib Zaidi is head of the PET Instrumentation \& Neuroimaging Laboratory at Geneva University Hospital and faculty member at the medical school of Geneva University. He is also a professor of medical physics at the University Medical Center of Groningen and adjunct professor of medical physics and molecular imaging at the University of Southern Denmark. He is actively involved in developing imaging solutions for cutting-edge interdisciplinary biomedical research and clinical diagnosis in addition to lecturing undergraduate and postgraduate courses on medical physics and medical imaging. His research is supported by the Swiss National Foundation (Total 4.35 M US\$) and centres on hybrid imaging instrumentation (PET/CT and PET/MRI), modeling medical imaging systems using the Monte Carlo method, development of computational anatomical models and radiation dosimetry, image correction, reconstruction, quantification, and kinetic 
modeling techniques in emission tomography as well as statistical image analysis in molecular brain and oncologic imaging, and, more recently, on novel design of dedicated PET and PET/MRI scanners. He was guest editor for nine special issues of peer-reviewed journals dedicated to medical image segmentation, PET instrumentation and novel quantitative techniques, computational anthropomorphic anatomical models, respiratory and cardiac gating in PET imaging, evolving medical imaging techniques, trends in PET quantification (two parts), PET/MR instrumentation and quantitative procedures, and nuclear medicine physics and instrumentation and serves as past editor-in-chief for the Open Medical Imaging Journal, deputy editor for the British Journal of Radiology, associate editor for Medical Physics, the International Journal of Biomedical Imaging, the International Journal of Tomography \& Simulation, and the Journal of Engineering \& Applied Sciences. He is also a member of the editorial board of Nuclear Medicine Communications, Clinical and Translational Imaging, American Journal of Nuclear Medicine and Molecular Imaging, International Journal of Molecular Imaging, Biomedical Imaging and Intervention Journal, the Open Medical Imaging Journal, the Open Neuroimaging Journal, International Journal of Biomedical Engineering, Consumer Health Informatics, and Medical Physics Journal and serves as scientific reviewer for leading journals in medical imaging. He has been elected as senior member of the IEEE and liaison representative of the International Organization for Medical Physics (IOMP) to the World Health Organization (WHO) in addition to being affiliated with several international medical physics and nuclear medicine organizations. He is involved in the evaluation of research proposals for European and International granting organizations and participates in the organization of international symposia and top conferences as member of scientific committees. His academic accomplishments in the area of quantitative PET imaging have been well recognized by his peers and by the medical imaging community at large because he is a recipient of many awards and distinctions, among which the prestigious 2003 Young Investigator Medical Imaging Science Award given by the Nuclear Medical and Imaging Sciences Technical Committee of the IEEE, the 2004 Mark Tetalman Memorial Award given by the Society of Nuclear Medicine, the 2007 Young Scientist Prize in Biological Physics given by the International Union of Pure and Applied Physics (IUPAP), the prestigious (100’000\$) 2010 Kuwait Prize of Applied Sciences (known as the Middle Eastern Nobel Prize) given by the Kuwait Foundation for the Advancement of Sciences (KFAS) for "outstanding accomplishments in Biomedical technology," the 2013 John S. Laughlin Young Scientist Award given by the American Association of Physicists in Medicine (AAPM), the 2013 Vikram Sarabhai Oration Award given by the Society of Nuclear Medicine, India (SNMI), and the 2015 Sir Godfrey Hounsfield Award given by the British Institute of Radiology (BIR). Dr. Zaidi has been an invited speaker of over 110 keynote lectures and talks at an International level, has authored over 440 publications, including 208 peer-reviewed journal articles (ISI-h index $=32-$ Web of Science $^{\mathrm{TM}},>7^{\prime} 110+$ citations - Google Scholar), conference proceedings and book chapters and is the editor of three textbooks on therapeutic applications of Monte Carlo calculations in nuclear medicine, quantitative analysis in nuclear medicine imaging, and molecular imaging of small animals.

\section{References}

\section{No reference}

